\title{
The Role and Views of Ophthalmologists During the COVID-I 9 Pandemic
}

\author{
Friederike C Kortuem ${ }^{1, *}$ \\ Focke Ziemssen $\mathbb{D}^{1,2, *}$ \\ Karsten U Kortuem ${ }^{3}$ \\ Constanze Kortuem' \\ 'University Eye Hospital, Center for \\ Ophthalmology, University of Tübingen, \\ Tübingen, Germany; ${ }^{2}$ Department of \\ Ophthalmology, University Hospital \\ Leipzig, Leipzig, Germany; ${ }^{3}$ University Eye \\ Hospital Ulm, University of Ulm, Ulm, \\ Germany
}

*These authors contributed equally to this work
Correspondence: Focke Ziemssen Department of Ophthalmology, University Hospital Leipzig, Liebigstr. 12, Leipzig, 04103, Germany

Tel +493419721650

Fax +49 3419721659

Email focke.ziemssen@med.uni-

tuebingen.de
Purpose: The severe acute respiratory syndrome coronavirus 2 (SARS-CoV-2) pandemic forced ophthalmologists to adjust their working conditions to ensure patient and staff safety, while still providing effective and timely treatment. This international survey among ophthalmologists was initiated to capture what actions ophthalmologists were taking and what their opinions were on the risks of infection in their workplace, the delay in treatment, the use of telemedicine and telephone for appointments, and the regional specifications and measures implemented by the respective authorities.

Methods: An open-source web tool was used to develop an online survey, to which ophthalmologists worldwide were invited via e-mail using international mailing lists (Media Mice, Singapore; Texere Publishing Inc, USA; CGO Gerling) and incentivized using a lottery. The physicians provided their level of agreement relating to the offered statements and gave free answers to the questions regarding the actions taken (conducted November 5th 2020 to December 20th 2020).

Results: After 91,000 invitations, responses were collected from 1122 ophthalmologists. Despite the use of large international mailing lists, mainly doctors from Europe participated. Half of the participants expressed great concern about possible SARS-CoV-2 infection in their patients. A significant number of younger ophthalmologists ( $\leq 50$ years: $76.9 \%, n=313$; $>50$ years: $69.6 \%, \mathrm{n}=181$ ) feared the delays that COVID-19 could cause to treatment. Reductions in patient numbers were broadly observed, with more ophthalmologists of younger age reporting greater declines. Nearly all ophthalmologists indicated that they provided disinfectant and the majority also used masks and questionnaires for screening. For $60.3 \%(n=412)$ of ophthalmologists, telephone calls reduced the risk of 'no-shows'; $71.6 \%(n=497)$ disagreed that telemedical evaluation is possible without slit lamp findings and fundus photos; and $57.0 \%$ of participants felt content with the governmental measures during the COVID-19 pandemic.

Conclusion: The COVID-19 pandemic has significantly influenced the work of ophthalmologists. Based on the limited response rate, certain statements were only possible to evaluate for the European Union: with a noticeable reduction in patient numbers, delay in treatment was a major worry. Measures to protect and reassure patients should be undertaken, especially regarding those with vision-threatening diseases requiring treatment.

Keywords: COVID-19, survey, infection control, occupational safety

\section{Introduction}

Due to the nature of many ophthalmological examinations, close patient contact is often required, putting ophthalmologists and patients at increased risk of acquiring severe acute respiratory syndrome coronavirus 2 (SARS-CoV-2) during the current pandemic. ${ }^{1,2}$ The ophthalmologist Li Wenliang from Wuhan was one of the first to 
warn of COVID-19 and himself died at the age of 33 due to his infection with the virus. ${ }^{3}$ Patients fear infection, which has been measured by the development of different scales to measure COVID-19-related anxiety. ${ }^{4,5}$ They also fear health status deterioration due to the lack of treatment. In a study conducted in Canada, $16 \%$ of the patients surveyed feared vision loss because of delays in treatment for their neovascular age-related macular degeneration (nAMD). ${ }^{6}$ Women and patients with less education worried significantly more and had a greater tendency to postpone treatment. Wearing a nose-mouth mask (NMM) is essential and effective in preventing infections. ${ }^{7-9}$ Meanwhile, public healthcare became more open to modern technologies, as telemedicine became widely reimbursable in both Europe and the United States.

We report on the results of an internationally conducted survey among ophthalmologists to assess the changes they have made to patient care and how they judge the risks of infection, delay of treatment, and the use of phone calls and telemedicine in providing care. Observed changes in patient numbers during the pandemic and the perceived effectiveness of governmental measurements in providing aid during the pandemic are also presented.

\section{Materials and Methods}

Some data of the survey, on examination lenses fogging, were recently pre-reported. ${ }^{10}$ The online survey was designed using an open-source web tool (LimeSurvey Version 3.24.3, Germany). Most questions consisted of a five-point Likert scale and were evaluated in a pilot phase with 20 colleagues.

The data collection took place between 5th of November and 20th of December 2020, and the survey was advertised using various international platforms (Media Mice, Singapore; Texere Publishing Inc, USA; CGO Gerling) via email. Of the 91,000 invitations sent out, the open rate of the individual commercial address distributors varied between 7.3 and $20.0 \%$ and the response rate between 0.8 and $1.4 \%$ (Figure 1).

Demographics such as age, gender and years of experience in ophthalmology were collected. On request, participants provided their level of agreement relating to statements on 1. infection; risk; 2 . delayed treatment; 3. utility of phone calls; 4. utility of telemedicine; and 5. measures taken by their government. The observed reduction in patients during the pandemic was recorded using a stepless slider. The participants had the option to describe their own measures for transmission prevention at their office or clinic and could indicate if and by how much they had observed a reduction in patient numbers.

The authors analyzed the data using SPSS (IBM SPSS Statistics Version 27.0.0.0, IBM Corporation, USA) for descriptive statistics. In addition, we used the chi-square test with the Yate's correction for continuity for the ordinal data and the Mann-Whitney $U$-test for the continuously distributed data. The Pearson correlation coefficient was used to test for correlation.

\section{Results}

\section{Participation and Demographics}

While 1122 participants fully or partially completed the survey, 685 ophthalmologists provided answers about adaptations to patient care, depending on the question (Supplementary Table 1). There was an even distribution of participants' ages across the decades of working life. There was no significant gender discrepancy among the responders. The majority of participating ophthalmologists had extensive professional experience (10 to 20 years: $24.7 \%, 20$ to 40 years: $39.7 \%$ ). Despite the large number of invitations across all continents (178 out of 195 countries), participation varied significantly by geographical location; the majority of participating physicians $(85.2 \%$, $\mathrm{n}=583$ ) practiced in Europe.

\section{Risk of Infection and Reduction in Patient Number per Week}

Nearly half of the ophthalmologists were concerned about virus transmission among patients during their treatment (Table 1). Ophthalmologists under 40 years of age and with under 10 years of experience were the most worried ( $\leq 40$ years: $57.0 \%, \mathrm{n}=139 ;>40$ years $43.0 \%, \mathrm{n}=189 ; \mathrm{p}=$ 0.005 and $\leq 10$ years: $60.1 \%, \mathrm{n}=134 ;>10$ years $42.1 \%$, $\mathrm{n}=194 ; \mathrm{p}=0.001)$. African ophthalmologists $(100 \%$, $\mathrm{n}=5)$ were more worried than those in Asia $(63.6 \%$, $\mathrm{n}=7)$, Europe $(44.9 \%, \mathrm{n}=262)$ or North America $(63.5 \% . n=47)$, with Europeans being the least worried. Due to the limited number of participants from Asia and Africa, the results of the continent-based analysis may be biased.

The majority of participating ophthalmologists $(73.9 \%$, $\mathrm{n}=505$ ) feared the consequences of delayed treatment due to the pandemic. There was no significant difference between ophthalmologists with different levels of experience or age. Again, European ophthalmologists were the least worried $(71.5 \%, \mathrm{n}=417)$, and those from Africa 


\section{Ophthalmology in times of SARS-CoV-2 pandemic}

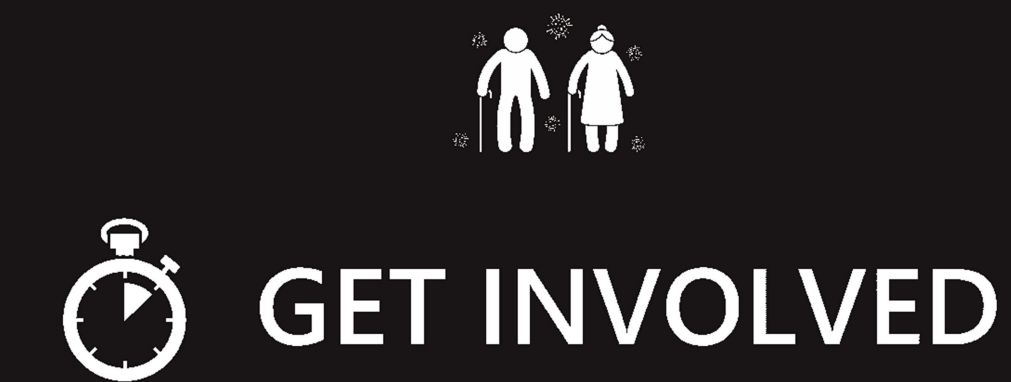

EXCHANGE ABOUT BEST PRACTICE AND PRACTICAL PEARLS

Take the chance to win a Volk lens in ony 5 minutes

\section{START SURVEY}

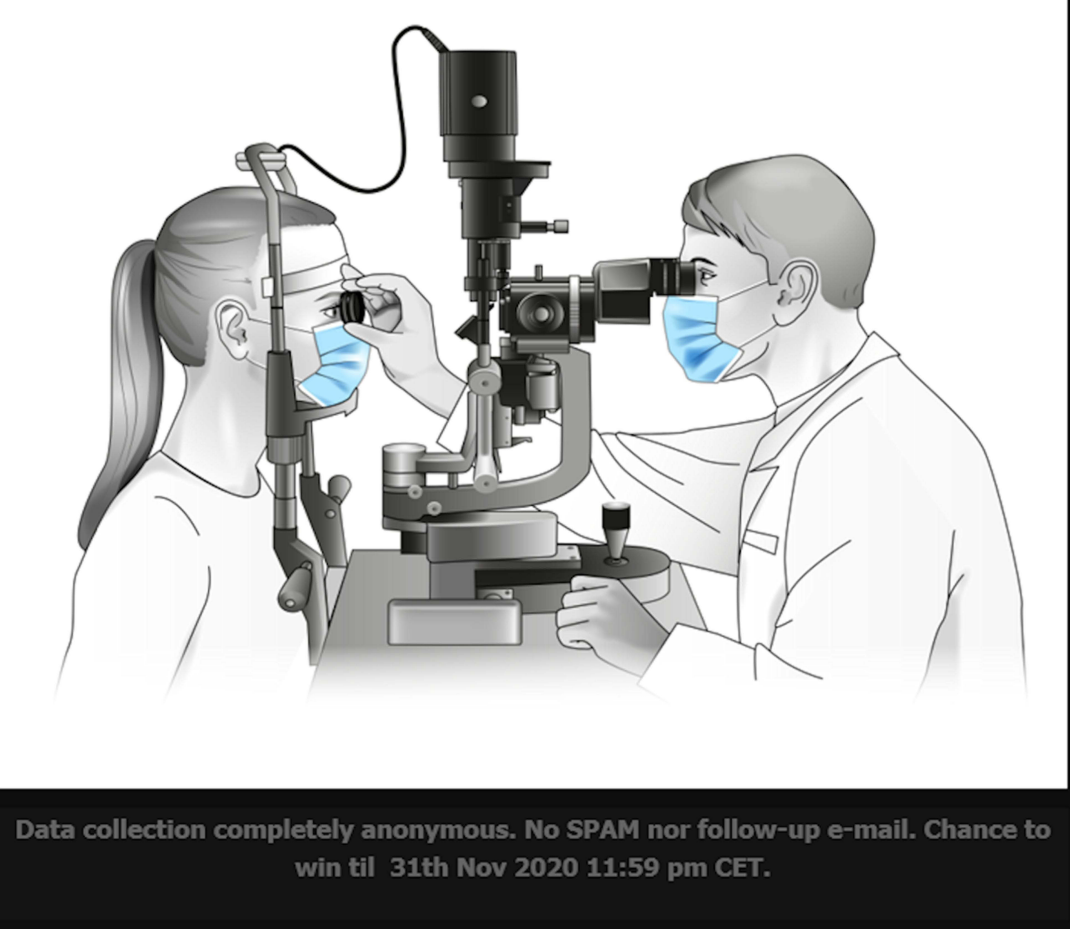

Figure I Example view of the responsive email template that invited participation in the survey. 


\begin{tabular}{|c|c|c|c|c|c|c|c|c|}
\hline & 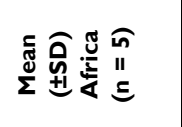 & 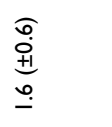 & 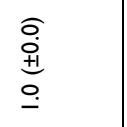 & 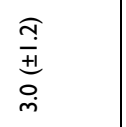 & $\begin{array}{l}\stackrel{0}{\stackrel{0}{0}} \\
\stackrel{0}{-}\end{array}$ & $\begin{array}{l}\stackrel{\widehat{m}}{=} \\
\stackrel{0}{N}\end{array}$ & 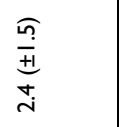 & $\begin{array}{l}\overline{=} \\
\stackrel{+}{+1} \\
\stackrel{\infty}{n}\end{array}$ \\
\hline & 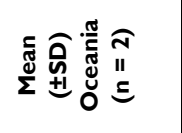 & 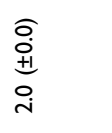 & $\begin{array}{l}\hat{0} \\
\text { ì } \\
\text { +1 } \\
\text { ì }\end{array}$ & $\begin{array}{l}\text { o. } \\
\text { ì } \\
\text { +1 } \\
\text { i }\end{array}$ & 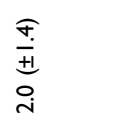 & 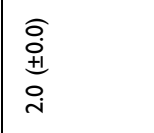 & 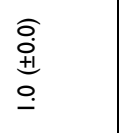 & 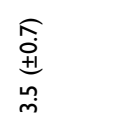 \\
\hline & 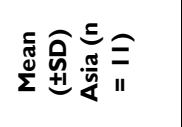 & 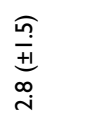 & $\begin{array}{l}\bar{T} \\
\bar{i} \\
\bar{i}\end{array}$ & 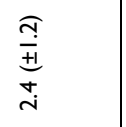 & 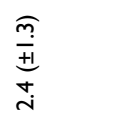 & 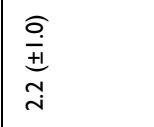 & $\begin{array}{l}\underset{F}{T} \\
\underset{+1}{+1} \\
\stackrel{i}{i}\end{array}$ & $\begin{array}{l}\overline{\bar{i}} \\
\text { +it } \\
\hat{m}\end{array}$ \\
\hline & 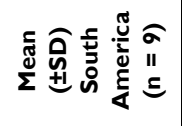 & 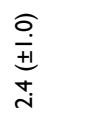 & 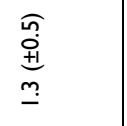 & 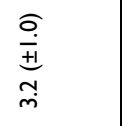 & 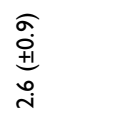 & $\begin{array}{l}\frac{a}{0} \\
\stackrel{+}{+1} \\
\stackrel{+}{i}\end{array}$ & 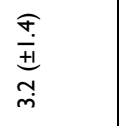 & $\begin{array}{l}\frac{\pi}{+} \\
\stackrel{+1}{+1} \\
\infty \\
\dot{m}\end{array}$ \\
\hline & 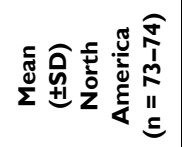 & 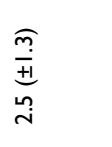 & $\begin{array}{l}a \\
\text { ô } \\
\text { +1 } \\
\end{array}$ & 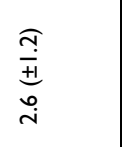 & $\begin{array}{l}\frac{a}{\dot{+}} \\
\stackrel{+1}{n} \\
\text { vi }\end{array}$ & $\begin{array}{l}\frac{a}{\rho+} \\
\stackrel{+1}{+} \\
\text { vi }\end{array}$ & $\begin{array}{l}\frac{0}{m} \\
\frac{m}{+1} \\
o \\
\dot{+}\end{array}$ & 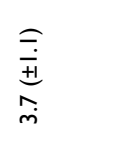 \\
\hline & 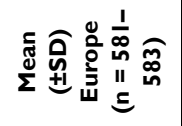 & $\begin{array}{l}\frac{\pi}{T} \\
\stackrel{+}{+1} \\
\text { S̀ }\end{array}$ & $\begin{array}{l}\frac{1}{a} \\
\text { à } \\
\text { an }\end{array}$ & 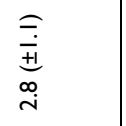 & $\begin{array}{l}\sigma \\
\sigma \\
0 \\
+1 \\
+ \\
\dot{i}\end{array}$ & 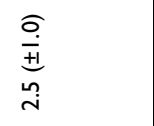 & $\begin{array}{l}\overline{\overline{+1}} \\
\substack{+1 \\
\text { ñ }}\end{array}$ & $\begin{array}{l}\sigma \\
\sigma \\
\dot{+} \\
+1 \\
\sigma \\
\dot{m}\end{array}$ \\
\hline & 部 & $\stackrel{\mathscr{o}}{\circ}$ & $\stackrel{\mathscr{0}}{\circ}$ & 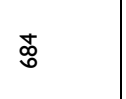 & 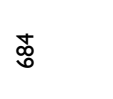 & $\mathscr{O}_{0}^{\infty}$ & ळొ & $\mathscr{\varrho}_{0}^{\infty}$ \\
\hline & 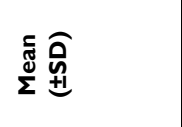 & $\begin{array}{l}\text { a } \\
\stackrel{+}{+1} \\
\sigma \\
\dot{i}\end{array}$ & $\begin{array}{l}\frac{\sigma}{+} \\
+1 \\
\bar{i}\end{array}$ & $\begin{array}{l}\overline{\bar{i}} \\
\stackrel{+1}{+1} \\
\infty \\
\stackrel{\sim}{i}\end{array}$ & $\begin{array}{l}a \\
\hat{O} \\
+1 \\
+ \\
\dot{i}\end{array}$ & 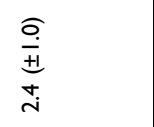 & $\begin{array}{l}\frac{\pi}{i} \\
+1 \\
\hat{i}\end{array}$ & 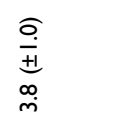 \\
\hline & 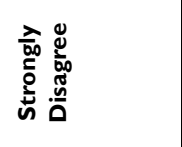 & $\begin{array}{l}\frac{\widehat{\sigma}}{\bar{a}} \\
\stackrel{0}{0} \\
\frac{0}{0}\end{array}$ & 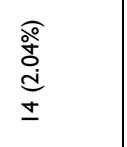 & 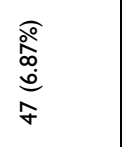 & 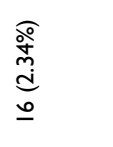 & 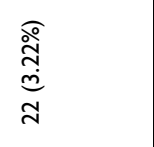 & 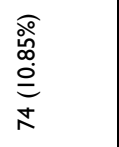 & 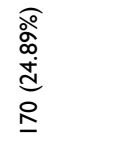 \\
\hline & 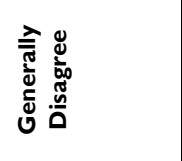 & 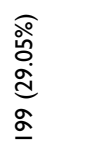 & 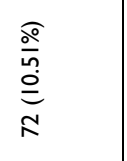 & 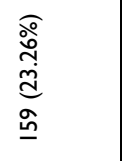 & 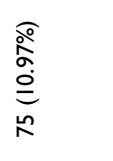 & 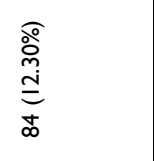 & 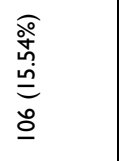 & 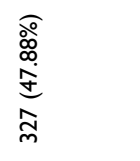 \\
\hline 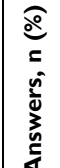 & 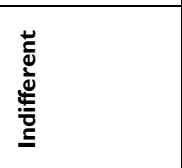 & 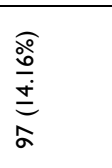 & & 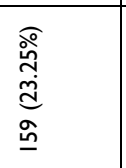 & 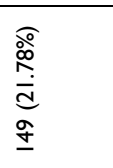 & 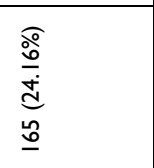 & 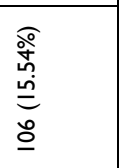 & $\begin{array}{l}\text { ळ } \\
\stackrel{0}{+} \\
\stackrel{+}{=} \\
\stackrel{0}{0}\end{array}$ \\
\hline & 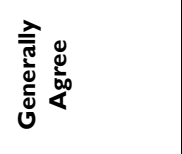 & 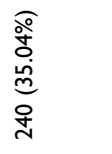 & 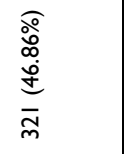 & 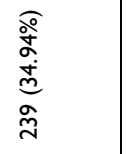 & 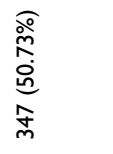 & 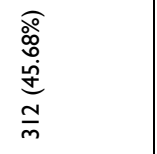 & 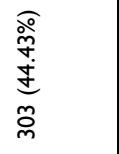 & 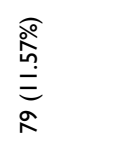 \\
\hline & 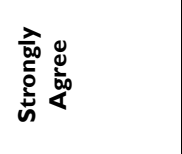 & & $\begin{array}{l}\text { o̊ } \\
\stackrel{0}{\grave{d}} \\
\text { do } \\
\underline{\alpha}\end{array}$ & 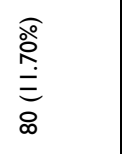 & 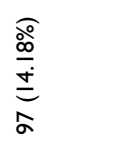 & 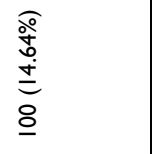 & 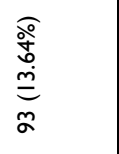 & 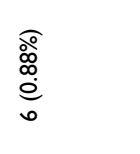 \\
\hline 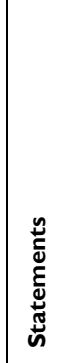 & & 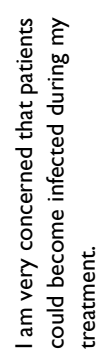 & 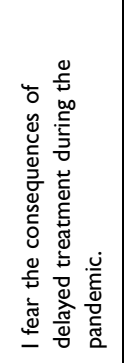 & 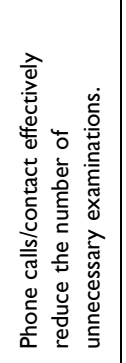 & 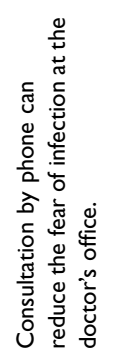 & 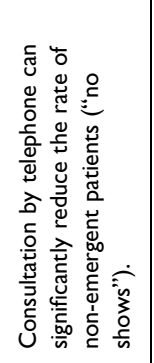 & 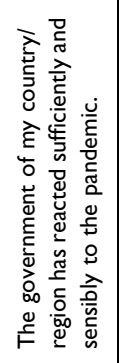 & 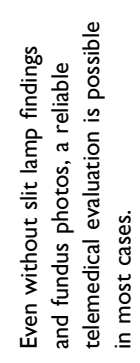 \\
\hline
\end{tabular}


Table 2 Patients Seen Before the Pandemic (Categories: I: <50; 2: 50-100; 3: 10I-150; 4: 15I-200; 5: >200) and Perceived Mean Reduction Due to the COVID-19 Pandemic Depending on Continent, Gender, Age and Experience

\begin{tabular}{|c|c|c|}
\hline & Mean Patients per Week & Mean Reduction in Patients $\mathbf{I S D}$ \\
\hline \multicolumn{3}{|l|}{ Continent } \\
\hline Europe & $3.2 \pm 1.3(n=583)$ & $20.1 \pm 17.6(n=583)$ \\
\hline North America & $2.1 \pm 0.9(n=74)$ & $32.6 \pm 20.4(n=74)$ \\
\hline South America & $2.4 \pm 0.7(n=9)$ & $32.2 \pm 15.4(n=9)$ \\
\hline Asia & $3.0 \pm 1.7(n=11)$ & $33.9 \pm 18.7(n=11)$ \\
\hline Africa & $2.2 \pm 0.8(n=5)$ & $43.0 \pm 17.9(n=5)$ \\
\hline Oceania & $2.0 \pm 0(n=2)$ & $17.5 \pm 17.7(n=2)$ \\
\hline \multicolumn{3}{|l|}{ Gender } \\
\hline Female & $3.0 \pm 1.2(n=351)$ & $21.2 \pm 18(n=351)$ \\
\hline Male & $3.1 \pm 1.3(n=331)$ & $22.9 \pm 18.8(n=332)$ \\
\hline \multicolumn{3}{|l|}{ Age } \\
\hline Age not indicated & $2.7 \pm 1.4(n=17)$ & $25.2 \pm 15.8(n=17)$ \\
\hline Age $21-30$ & $2.5 \pm 1.0(n=66)$ & $18.0 \pm 14.4(n=66)$ \\
\hline Age $31-40$ & $2.9 \pm 1.2(n=178)$ & $20.7 \pm 18.1(n=178)$ \\
\hline Age $4 I-50$ & $3.0 \pm 1.3(n=162)$ & $23.9 \pm 20.8(n=163)$ \\
\hline Age $5 I-60$ & $3.4 \pm 1.3(n=174)$ & $21.7 \pm 18.0(n=174)$ \\
\hline Age $>60$ & $3.1 \pm 1.3(n=86)$ & $24.2 \pm 18.3(n=84)$ \\
\hline \multicolumn{3}{|l|}{ Experience } \\
\hline Less than 5 years & $2.5 \pm 1.1(n=113)$ & $20.3 \pm 17.2(n=113)$ \\
\hline $5-10$ years & $2.9 \pm 1.2(n=109)$ & $21.0 \pm 17.8(n=110)$ \\
\hline $10-20$ years & $3.1 \pm 1.3(n=169)$ & $21.9 \pm 18.8(n=169)$ \\
\hline $20-40$ years & $3.3 \pm 1.3(n=27 I)$ & $23.11 \pm 19.1(n=271)$ \\
\hline Over 40 years & $2.7 \pm 1.3(n=21)$ & $22.3 \pm 18.6(n=21)$ \\
\hline
\end{tabular}

$(100 \%, \mathrm{n}=5)$ the most. Fear of transmission correlated significantly with the fear of delayed treatment (Pearson's $\mathrm{r}=0.29, \mathrm{p}<0.001)$.

The majority of participating ophthalmologists saw either $50-100(29.6 \%)$ or $101-150(24.2 \%)$ patients per week before the pandemic. Approximately 18\% saw 151200 and another $18 \%$ over 200 patients per week. Younger and less experienced ophthalmologists generally saw fewer patients (Table 2). The largest group, the European ophthalmologists, reported the highest number of patients per week (approximately 25\%: 50-100 or 100-150 patients per week, and approximately 20\%: 151-200 or over 200 patients per week; Table 2). In comparison, in the second largest group, ophthalmologists from North America, 52.7\% reported examining 50-100 patients per week. There was no significant difference between genders (Table 2). Eleven percent of participating ophthalmologists $(n=75)$ reported no change in patient numbers. The mean reduction in patient numbers during the pandemic was
22.0\% (CI 20.6-23.4\%, $\mathrm{n}=683$ ). Ophthalmologists older than 40 years old $(\mathrm{p}=0.025$; $\leq 40$ years: $20.0 \% \mathrm{n}=244$; $>40$ years: $23.1 \%, \mathrm{n}=440)$ and colleagues who saw under 100 patients per week ( $\mathrm{p}=0.005 ; \leq 100$ patients: $24.8 \%$, $\mathrm{n}=276 ;>100$ patients: $20.5 \%, \mathrm{n}=407$ ) before the pandemic observed a significantly greater reduction in numbers.

\section{Protective Measures and Specifications by the Authorities}

In total, 655 participants $(94.2 \%)$ indicated that they had dispensers with disinfectant at the entrance to their places of practice; 517 (74.4\%) provided masks; 274 (39.4\%) measured the patient's temperature upon arrival; 393 $(56.5 \%)$ used questionnaires to screen for potentially infected patients; and 93 (13.4\%) indicated that they take additional measures (see Supplementary Table 2).

The spectacles wearers among the ophthalmologists were slightly older (33.6\%: up till 40 years; $42.5 \%$ above 
Table 3 Results of Scale of Satisfaction with the Respective Governments of Different Countries (Only Countries with at Least 10 Answers are Shown) and Their Deaths per Million Capita Due to SARS-CoV-2 Infection Cited on the 15th of July 202I (Source Statista 'I); Strongly Agree: I; Generally Agree: 2 Indifferent: 3; Generally Disagree: 4; Strongly Disagree: 5

\begin{tabular}{|l|c|c|}
\hline Country & Mean \pm SD Satisfaction with Government & Deaths per I Million Capita \\
\hline Austria $(n=22)$ & $2.6 \pm 0.9$ & 1201. I \\
Germany $(n=456)$ & $2.4 \pm 1.0$ & 1081.9 \\
Italy $(n=10)$ & $3.5 \pm 1.6$ & 2106.8 \\
Mexico $(n=49)$ & $4.3 \pm 1.1$ & 1804.3 \\
Switzerland ( $=17)$ & $2.9 \pm 1.1$ & 1266.6 \\
UK $(n=15)$ & $2.8 \pm 1.2$ & 1909.8 \\
USA $(n=23)$ & $3.5 \pm 1.5$ & 1819.5 \\
\hline
\end{tabular}

40 years, Supplementary Table 3 ). There was no difference in the assessment of the risk of infection between those who did and did not wear spectacles.

One third of the participants $(n=239)$ generally agreed that phone contact with patients effectively reduced the number of unnecessary examinations, but $23.3 \%(n=159)$ disagreed or were indifferent on the topic. Here, ophthalmologists under 40 years of age ( $\leq 40$ years: $53.3 \% \mathrm{n}=$ 130 ; $>40$ years: $43.1 \%, \mathrm{n}=189$ ) and with under 10 years' experience $(\leq 10$ years: $52.9 \%, n=118 ;>10$ years $43.7 \%$, $\mathrm{n}=201)$ were significantly more likely to agree $(\mathrm{p}=0.01)$. Nearly two thirds $(65.0 \%, \mathrm{n}=440)$ claimed that consultation by phone could reduce the fear of infection at the doctor's office. There was no significant difference between younger and older or less and more experienced ophthalmologists. Also, for the majority (59.3\%, $n=412)$, it was an effective way of reducing the rate of nonemergent patients, especially for ophthalmologists under 40 years of age and those who were less experienced (both $\mathrm{p}<0.001)$, while $24.2 \%(\mathrm{n}=165)$ were indifferent.

Of the participants, $71.6 \%(n=497)$ did not agree that telemedical evaluation is possible without slit lamp findings and fundus photos, and $14.8 \%(\mathrm{n}=101)$ were indifferent, perhaps because they had not tried it. Regarding remote consultation without image information available, the assessment (agreement vs disagreement) was the same between younger and older or less and more experienced ophthalmologists. The agreement that telephone calls can reduce non-emergent patients correlated inversely with the reduction in patient numbers (Pearson's $\mathrm{r}=-0.10, \mathrm{p}=0.008$ ) and correlated with the agreement that telemedicine is effective even without slit lamp and fundus findings (Pearson's $r=0.24, \mathrm{p}<0.001$ ).

Over half $(57.0 \%, \mathrm{n}=396)$ of participants felt that their country or region's authorities had reacted sufficiently and sensibly to the pandemic, and $15.6 \%$ were indifferent $(\mathrm{n}=$
106). There was no significant difference between younger and older ophthalmologists. Knowing that the number of responses was very low for most of the regions, only countries with more than 10 participating ophthalmologists were considered (Table 3). In Germany, ophthalmologists were most pleased with their region's response (mean 2.4 \pm 1.0 ), and in Mexico they were the least pleased (mean $4.3 \pm 1.1$ ). For Germany, Austria, Switzerland, the UK and Italy, the satisfaction with the authorities correlated inversely with deaths per million capita due to COVID-19. Surprisingly, fear of patients getting infected at the office did not correlate with the satisfaction with the authorities' response (Pearson's $r=-0.008, p=0.84$ ).

\section{Discussion}

Patients and doctors were both required to follow the governmental guidelines of social distancing due to the emerging COVID-19 pandemic in the second quarter of 2020. In-office patient numbers subsequently reduced significantly and telemedicine thrived. ${ }^{12-14}$

Ophthalmologists have had to adapt their working environments to prevent virus transmission while providing predominantly face-to-face care. In the meantime, numerous survey results from different continents are available to provide further information. ${ }^{15,16}$ Although the email distribution list for this survey used very large distribution lists covering a number of continents, the response rate was mostly very low. For future studies, it may be more successful to use different channels, such as short messaging services and local representatives.

The levels of concern were higher in younger ophthalmologists, but almost all ophthalmologists worldwide who participated in our survey have undertaken measures to prevent the spread of infection. It is known that virus transmission via the ocular surface is possible and 
therefore any close work with the eye warrants extra caution. ${ }^{17}$ In addition, in low-income countries, patients were provided with disinfectants. ${ }^{18,19}$ Recommendations for the ophthalmological sector included intensive cleaning of instruments (slit lamps and lenses) after each patient ${ }^{20}$ and avoidance of on-contact tonometry ${ }^{21}$ because of the possibility of aerosolisation of virus particles. In order to classify the results correctly, the timing of the studies and the large regional differences in infection numbers must be taken into account, in addition to the low response rates, which mean that the results can not be considered representative for most countries.

Ophthalmologists older than 40 years old and those who saw under 100 patients per week before the pandemic observed a significantly greater reduction in patient numbers. However, preventative measures are crucial regardless of patient numbers. Since a major reduction in patient visits per week could also delay necessary treatment, adaptation to the pandemic was critical. ${ }^{22-24}$ Those whose patient numbers declined more dramatically were more worried than their colleagues about the effects that delayed treatment might have on their patients. It cannot be verified with certainty whether these are true differences, or whether doctors who were more concerned perceived and assessed both patient numbers and predicted effects differently. However, the influence on decreasing treatment numbers and frightened patients has been reported and analysed, ${ }^{25}$ fluctuated during the different phases of the pandemic and changed further after the vaccinations began.

Depending on the number of patients per ophthalmologist per week, there was no disparity regarding the level of concern about virus spread. Other surveys showed that a large proportion of ophthalmologists had no exposure to COVID19 patients. ${ }^{16}$ Many patients might have made the decision themselves not to seek treatment. In addition, the numbers varied greatly, both in different phases of the pandemic and regionally. Within our study, the doctors who saw more patients per week were more confident in the efficacy of their prevention measures. When the fear of delaying treatment precludes a reduction in patient visits, sensible solutions to allow patients to maintain distance, such as the use of pagers, spaced seating, and changes in workflow are essential in limiting the risk of transmission. ${ }^{26}$ Another effective solution could be to offer longer office hours with staff working in staggered shifts.

Participants reported that telephone calls were more effective in easing the patients' worries and avoiding noshows than in assessing the patients' symptoms for the diagnosis of a disease, the activity of a chronic disease, necessary treatment, or the need for the patients to have an in-person appointment. ${ }^{27-30} \mathrm{~A}$ telemedical evaluation with fundus photos or slit lamp findings is not yet broadly available to the public, but in some countries efforts are more advanced. For example, investigation units in the community (IUCs), ${ }^{31}$ which are examination facilities staffed by ophthalmic technicians, can relieve the burden for clinics frequently visited by chronically ill patients who require follow-up exams. Other studies have shown that telemedicine in ophthalmology is mainly used for external eye diseases or for diagnoses that can often be made from symptoms alone, such as allergic conjunctivitis. ${ }^{32}$

Contacting patients before their visit to ensure that they do not worry about infection, are well-informed, and attend their appointment is a difficult service to provide in a practical working environment. General information and education about safe behavior through the media may be more cost effective. On the other hand, it may be challenging for patients to filter out widespread misinformation, so a trusting relationship between doctor and patient is important. One solution might be to filter the appointment list for critical patients and keep appointments free for emergency cases. Reports from other countries also suggest that such changes will have a lasting impact on future treatment pathways. ${ }^{16,33-36}$

This survey found that ophthalmologists under 40 years old seemed to be the most willing to adapt their workplace practices to meet the challenges of COVID-19, took the threat of viral spread among their patients the most seriously, and suffered the greatest reduction in patient numbers during the pandemic in order to protect their patients. We must ensure that these young ophthalmologists stay satisfied with their work. ${ }^{37}$ At the same time, physicians are concerned about their own health and risk of infection, causing additional stress. ${ }^{38,39}$ Because of the close distance required, ophthalmologists feel that they are at greater risk than other specialists and are mostly open to vaccination. ${ }^{40,41}$

The population has suffered greatly during the COVID-19 pandemic and will continue to feel the effects with regards to the economy, education, employment and social well-being for a long time. Differing levels of concern between the different continents are most likely due to the different experiences during past pandemics. Due to the SARS-CoV-1 epidemic of 2002/2003, which mainly affected Asia, Asian people seem to be less worried about the current pandemic. ${ }^{42}$ Additionally, in poorer countries where resources for (self-)protection are limited, and 
vaccination and the reduction of the spread of infection are less manageable, the level of fear may be higher. ${ }^{43,44}$

A limitation of this study that should be considered is the heterogeneous participation of ophthalmologists from different countries, with possibly non-representative responses. Despite sending out worldwide invitations to participate in the survey, the response rate was 1) very low overall, and 2) significantly different for the European countries compared to other countries. ${ }^{16}$ Furthermore, these responses are a snapshot between different regional waves of the pandemic, before the availability of vaccines. All measures, introduced in their practice or by the government, are likely to vary widely over time and between regions. Unlike other studies, this study looked unilaterally at ophthalmologists' perspectives. ${ }^{2,45,46}$ However, some aspects, including those related to screening, ${ }^{47}$ were not recorded.

\section{Conclusion}

The COVID-19 pandemic has significantly affected ophthalmologists worldwide. They have taken the pandemic seriously, taken responsibility, and provided effective and safe measures to provide healthcare while not delaying treatment. Still, due to the close conditions in a doctor's office, we cannot exclude that there is a small risk of infection, and ophthalmologists must be careful to provide all safety measures possible. Selective communication with critically ill patients via telephone may decrease fear before a visit at the office and at the same time avoid unnecessary examinations. The indications of possible differences between age groups and individual countries need to be considered further. It may be useful to coordinate the design of future approaches across different countries and shape these measures proactively after appropriate discussion. Times of change can be an opportunity to incorporate better treatment patterns and practices.

\section{Data Sharing Statement}

All relevant data are included in the article. The datasets used and/or analyzed during the present study are available from the corresponding author on reasonable request.

\section{Ethical Approval and Informed Consent}

In accordance with the requirements and in consultations with the local ethics committee of Eberhard Karl University Tübingen, there was no need to obtain any ethical approval in advance of the survey. Only anonymous data from voluntary participants were analyzed.

\section{Consent for Publication}

By participating, participants consented to the analysis of anonymous data.

\section{Acknowledgment}

The authors would like to thank Irina Stingl for support with the graphical representation of this survey.

\section{Funding}

The authors did not receive support from any organization for the submitted work. The work was funded without commercial support by the authors.

\section{Disclosure}

Focke Ziemssen has received speaker fees and consultation honoraria from Allergan/AbbVie, Alimera, Bayer, Boehringer-Ingelheim, Oxurion, NovoNordisk, and Roche/Genentech, and grants and personal fees from Novartis, but none of the topics were related to this survey. The authors declare that they have no other potential conflicts of interest related to this work.

\section{References}

1. Jun ISY, Hui KKO, Songbo PZ. Perspectives on coronavirus disease 2019 control measures for ophthalmology clinics based on a Singapore center experience. JAMA Ophthalmol. 2020;138:435-436. doi:10.1001/jamaophthalmol.2020.1288

2. Said RB. DOs and DON'Ts in Ophthalmology during the COVID-19 pandemic. Int J Clin Res. 2021;1(1):65-67. doi:10.38179/ijcr.v1i1.26

3. Green A. Li Wenliang. Lancet. 2020;395(10225):682. doi:10.1016/ S0140-6736(20)30382-2

4. Lee SA. Coronavirus Anxiety Scale: a brief mental health screener for COVID-19 related anxiety. Death Stud. 2020;44(7):393-401. doi:10.1080/07481187.2020.1748481

5. Ahorsu DK, et al. The fear of COVID-19 scale: development and initial validation. Int J Mental Health Addict. 2020;1-9. doi:10.1007/ s11469-020-00270-8

6. Rozon J-P, Hébert M, Bourgault S, et al. Fear associated with COVID-19 in patients with neovascular age-related macular degeneration. Clin Ophthalmol. 2021;15:1153. doi:10.2147/OPTH. S300239

7. Tirupathi R, Bharathidasan K, Palabindala V, Salim SA, Al-Tawfiq JA. Comprehensive review of mask utility and challenges during the COVID-19 pandemic. Infez Med. 2020;28:57-63.

8. Aytoğan H, Ayintap E, Yılmaz NÖ. Specifying the role of masks in reducing viral material on environmental surfaces of an ophthalmology examination room-reply. JAMA Ophthalmol. 2021;139(3):366. doi:10.1001/jamaophthalmol.2020.6568

9. Du Q, Zhang D, Hu W, et al. Nosocomial infection of COVID-19: a new challenge for healthcare professionals. Int J Mol Med. 2021;47 (4):1. doi:10.3892/ijmm.2021.4864 
10. Kortuem FC, Ziemssen F, Kortuem KU, Kortuem C. International survey on COVID-19 pandemic: personal protective measures during fundus examination. Acta Ophthalmol. 2021. doi:10.1111/aos.14904

11. Statista; 2021. Available from: https://www.statista.com/statistics/ 1104709/coronavirus-deaths-worldwide-per-million-inhabitants/. Accessed September 17, 2021.

12. Anderson TS, O’Donoghue AL, Dechen T, Herzig SJ, Stevens JP. Trends in telehealth and in-person transitional care management visits during the COVID-19 pandemic. J Am Geriatrics Soc. 2021. doi:10.1111/jgs.17329

13. Alexander GC, Tajanlangit M, Heyward J, et al. Use and content of primary care office-based vs telemedicine care visits during the COVID-19 pandemic in the US. JAMA Netw Open. 2020;3(10): e2021476-e2021476. doi:10.1001/jamanetworkopen.2020.21476

14. Rohe AM, Kostev K, Sesterhenn AM. Impact of the COVID-19 pandemic on consultations and diagnosis in ENT practices in Germany. Laryngo-Rhino-Otologie. 2021. doi:10.1055/a-1510-9686

15. Williams AM, Kalra G, Commiskey PW, et al. Ophthalmology practice during the coronavirus disease 2019 pandemic: the University of Pittsburgh experience in promoting clinic safety and embracing video visits. Ophthalmol Ther. 2020;9(3):1-9. doi:10.1007/s40123-02000255-9

16. Sanjay S, Leo SW, Au Eong KG. et al. Global ophthalmology practice patterns during COVID-19 pandemic and lockdown. Ophthalmic Epidemiol;2021. 1-12. doi:10.1080/09286586.2021.1934037

17. Kitazawa K, Deinhardt-Emmer S, Inomata T, Deshpande S, Sotozono C. The transmission of SARS-CoV-2 infection on the ocular surface and prevention strategies. Cells. 2021;10(4):796. doi:10.3390/cells 10040796

18. Chu DK, Akl EA, Duda S, et al. Physical distancing, face masks, and eye protection to prevent person-to-person transmission of SARS-CoV-2 and COVID-19: a systematic review and meta-analysis. Lancet. 2020;395(10242):1973-1987. doi:10.1016/ S0140-6736(20)31142-9

19. Golin AP, Choi D, Ghahary A. Hand sanitizers: a review of ingredients, mechanisms of action, modes of delivery, and efficacy against coronaviruses. Am J Infect Control. 2020;48(9):1062-1067. doi:10.1016/j.ajic.2020.06.182

20. Mannan R, Pruthi A, Sud R, Khanduja S. Slit lamp examination during COVID-19: where should the protective barrier be? Ind J Ophthalmol. 2021;69(2):376. doi:10.4103/ijo.IJO_2204_20

21. Hao W, et al. Quantitative evaluation of aerosol generation from non-contact tonometry and its correlation with tear film characteristics. Advan Ther. 2021;1-11.doi:10.1007/s12325-021-01740-8

22. Shalaby WS, Odayappan A, Venkatesh R, et al. The impact of COVID-19 on individuals across the spectrum of visual impairment. $\mathrm{Am}$ J Ophthalmol. 2021;227:53-65. doi:10.1016/j.ajo.2021.03.016

23. Rahimzadeh M, Muniraju R, Izadi S. Effect of COVID-19 pandemic on anti-VEGF treatment of medical retinal conditions. Physician. 2021;6:1-9.

24. de Gage SB, et al. Intravitreal anti-vascular endothelial growth factor use in France during the coronavirus disease 2019 pandemic. JAMA Ophthalmol. 2020;139(2):240.

25. Mantica G, Riccardi N, Terrone C, Gratarola A. Non-COVID-19 non-COVID-19 visits to emergency departments during the pandemic: the impact of fear. Public Health. 2020;183:40. doi:10.1016/ j.puhe.2020.04.046

26. Zeinalnezhad M, Chofreh AG, Goni FA, Klemeš JJ, Sari E. Simulation and improvement of patients' workflow in heart clinics during covid-19 pandemic using timed coloured petri nets. Int J Environ Res Publ Health. 2020;17(22):8577. doi:10.3390/ ijerph 17228577

27. Chen Y, Ismail R, Cheema MR, Ting DS, Masri I. Implementation of a new telephone triage system in ophthalmology emergency department during COVID-19 pandemic: clinical effectiveness, safety and patient satisfaction. Eye. 2021;1-3.doi:10.1038/s41433-021-01528-8
28. Ghazala FR, et al. Live teleophthalmology avoids escalation of referrals to secondary care during COVID-19 lockdown. Clin Exp Optometry. 2021;1-6. doi:10.1080/08164622.2021.1916383

29. Newman-Casey PA, De Lott L, Cho J, et al. Telehealth-based eye care during the COVID-19 pandemic: utilization, safety, and the patient experience. Am J Ophthalmol. 2021;230:234-242. doi:10.1016/j.ajo.2021.04.014

30. Li J-PO, Thomas AAP, Kilduff CLS, et al. Safety of video-based telemedicine compared to in-person triage in emergency ophthalmology during COVID-19. EClinical Med. 2021;34:100818. doi:10.1016/j.eclinm.2021.100818

31. Tham Y-C, Husain R, Teo KYC, et al. New digital models of care in ophthalmology, during and beyond the COVID-19 pandemic. $\mathrm{Br}$ J Ophthalmol. 2021. doi:10.1136/bjophthalmol-2020-317683

32. Portney DS, Zhu Z, Chen EM, et al. COVID-19 and utilization of teleophthalmology: trends and diagnoses (CUT Group). Ophthalmology. 2021. doi:10.1016/j.ophtha.2021.02.010

33. Wickham L, Hay G, Hamilton R, et al. The impact of COVID policies on acute ophthalmology services-experiences from Moorfields Eye Hospital NHS Foundation Trust. Eye (Lond). 2020;34:1189-1192. doi:10.1038/s41433-020-0957-2

34. Goel M, Goel S, Sachdev M, et al. Post-lockdown challenges for ophthalmologists during COVID-19 pandemic in India: a survey-based analysis. Ind $J$ Ophthalmol. 2021;69(4):946. doi:10.4103/ijo.IJO 355020

35. Ashour DM, et al. Challenges of personal protective equipment use among ophthalmologists during the COVID-19 pandemic: a multicenter international study. Eur $J$ Ophthalmol. 2021;25:11206721211028037.

36. Scott DA, Hadden PW, Wilson GA. Impact of the COVID-19 pandemic lockdown on public sector ophthalmic work by New Zealand's ophthalmologists. N Z Med J. 2021;134:120-127.

37. Alrawashdeh HM, Al-Tammemi AB, Alzawahreh MK, et al. Occupational burnout and job satisfaction among physicians in times of COVID-19 crisis: a convergent parallel mixed-method study. BMC Public Health. 2021;21(1):1-18. doi:10.1186/s12889021-10897-4

38. Magnavita N, Chirico F, Garbarino S, et al. SARS/MERS/SARSCoV-2 outbreaks and Burnout Syndrome among healthcare workers. An umbrella systematic review. Int J Environ Res Public Health. 2021;18(8):4361. doi:10.3390/ijerph18084361

39. Navarro V. Why Asian Countries are controlling the pandemic better than the United States and Western Europe. Int J Health Serv. 2021;51(2):261-264. doi:10.1177/0020731421999930

40. Kavadarlı I, Mutlu M. Effects of the COVID-19 pandemic on Turkish Ophthalmologists. Turkish J Ophthalmol. 2021;51(2):95. doi:10.4274/tjo.galenos.2020.52563

41. Roth M, Holtmann C, Tillmann A, Bertram B, Geerling G. Assessment of subjective risk of infection and willingness to vaccinate against SARS-CoV-2 among German ophthalmologists: results of a survey by DOG and BVA. Der Ophthalmologe: Zeitschrift der Deutschen Ophthalmologischen Gesellschaft. 2021. doi:10.1007/ s00347-021-01425-1

42. Konopińska J, Obuchowska I, Lisowski Ł, et al. Intention to get COVID-19 vaccinations among ophthalmology residents in Poland: a cross-sectional survey. Vaccines. 2021;9(4):371. doi:10.3390/ vaccines 9040371

43. Solís Arce JS, Warren SS, Meriggi NF, et al. COVID-19 vaccine acceptance and hesitancy in low- and middle-income countries. Nat Med. 2021;27(8):1385-1394. doi:10.1038/s41591-021-01454-y

44. Nachega JB, Grimwood A, Mahomed H, et al. From easing lockdowns to scaling up community-based coronavirus disease 2019 screening, testing, and contact tracing in Africa-shared approaches, innovations, and challenges to minimize morbidity and mortality. Clin Infect Dis. 2021;72(2):327-331. doi:10.1093/cid/ciaa695 
45. Dar S, De Moraes CG, Karani R, et al. Patient concerns regarding suspended ophthalmic care due to COVID-19. J Glaucoma. 2021;30 (8):750-757. doi:10.1097/IJG.0000000000001877

46. Teo CL, Chee ML, Koh KH, et al. COVID-19 awareness, knowledge and perception towards digital health in an urban multi-ethnic Asian population. Sci Rep. 2021;11(1):1-13. doi:10.1038/s41598-02190098-6
47. Kumar K, Sampritha UC, Maganty V, et al. Pre-operative SARS $\mathrm{CoV}-2$ rapid antigen test and reverse transcription polymerase chain reaction: a conundrum in surgical decision making. Ind J Ophthalmol. 2021;69(6):1560-1562. doi:10.4103/ijo.IJO_430_21

\section{Publish your work in this journal}

Clinical Ophthalmology is an international, peer-reviewed journal covering all subspecialties within ophthalmology. Key topics include: Optometry; Visual science; Pharmacology and drug therapy in eye diseases; Basic Sciences; Primary and Secondary eye care; Patient Safety and Quality of Care Improvements. This journal is indexed on PubMed

Submit your manuscript here: https://www.dovepress.com/clinical-ophthalmology-journal
Central and CAS, and is the official journal of The Society of Clinical Ophthalmology (SCO). The manuscript management system is completely online and includes a very quick and fair peer-review system, which is all easy to use. Visit http://www.dovepress.com/ testimonials.php to read real quotes from published authors. 